\title{
Optical Responses of Localized and Extended Modes in a Mesoporous Layer on Plasmonic Array to Isopropanol Vapor
}

\author{
Shunsuke Murai,* Elena Cabello-Olmo, Ryosuke Kamakura, Mauricio E. Calvo, Gabriel Lozano,* \\ Taisuke Atsumi, Hernán Míguez, and Katsuhisa Tanaka
}

Cite This: https://dx.doi.org/10.1021/acs.jpcc.9b10999

Read Online

ACCESS | Lill Metrics \& More | 国 Article Recommendations | st Supporting Information

\begin{abstract}
Mesoporous silica features open and accessible pores that can intake substances from the outside. The combination of mesoporous silica with plasmonic nanostructures represents an interesting platform for an optical sensor based on the dependence of plasmonic modes on the refractive index of the medium in which metallic nanoparticles are embedded. However, so far only a limited number of plasmonic nanostructures are combined with mesoporous silica, including random dispersion of metallic nanoparticles and flat metallic thin films. In this study, we make a mesoporous silica layer on an aluminum nanocylinder array. Such plasmonic arrangements support both localized surface plasmon resonances (LSPRs) and extended modes which are the result of the hybridization of LSPRs and photonic modes

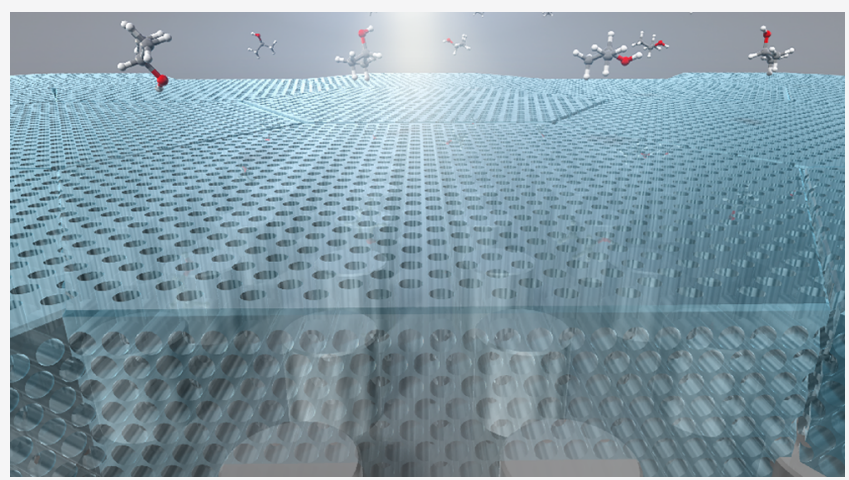
extending into the mesoporous layer. We investigate in situ optical reflectance of this system under controlled pressure of isopropanol vapor. Upon exposure, the capillary condensation in the mesopores results in a gradual spectral shift of the reflectance. Our analysis demonstrates that such shifts depend largely on the nature of the modes; that is, the extended modes show larger shifts compared to localized ones. Our materials represent a useful platform for the field of environmental sensing.
\end{abstract}

\section{INTRODUCTION}

Porous photonic structures are a class of resonant materials where pores or voids play a critical role in their optical properties. ${ }^{1}$ Open porosity accessible from the outside can be used as a room to store materials to enhance/modulate the optical response. In particular, magnetic, ${ }^{2}$ plasmonic, ${ }^{3,4}$ luminescent, ${ }^{5}$ and phase transition ${ }^{6-8}$ materials have been incorporated into pores. Also, it allows porous photonic structures to respond to changes in the surrounding environment, as it has been demonstrated in one- ${ }^{9-12}$ and threedimensional $^{13-15}$ photonic crystals, where a shift in the optical response is observed as a result of vapor adsorption.

Plasmonic nanostructures are another class of systems that respond to the environment: The excitation condition of a surface plasmon polariton (SPP), a plasma oscillation on the metallic surface coupled to a lightwave, ${ }^{16}$ varies with the refractive index of the surroundings. Thus, adsorption of molecules on the surface of metallic nanostructures may result in the spectral shift in resonant condition. Chemical- and antibody-modified metallic thin films ${ }^{17,18}$ and nanoparticles ${ }^{19-21}$ are well-studied examples to detect target molecules in solution. For the vapor and gas sensing, there are also SPPenhanced sensing systems. $^{22-29}$

A combination of porous materials with advanced plasmonic nanostructures should make a unique system that shows environmental sensing ability. In particular, mesoporous silica (MPS) represents an interesting choice because the mesoscale pores do not scatter visible light to smear out the plasmonic resonances, although there is only limited number of examples that combine mesoporous materials with plasmonic thin films $s^{30}$ and nanostructures. A periodic plasmonic array, where $\mathrm{Al}$ nanocylinders are arranged in a period comparable to the wavelength of light, ${ }^{31}$ supports localized surface plasmon resonances (LSPRs). In addition, it could support the hybrid modes; that is, the coherent in-plane light scattering results in the strong radiative coupling between LSPRs. Covering such an array with a layer of transparent MPS makes a system where light energy is trapped inside the layer with accessible pores. Although it is known that optical modes sustained by such systems can be tuned by the refractive index of the layer deposited atop, ${ }^{31}$ an in situ response of such a plasmonicphotonic hybrid structure has never been explored.

In this study, we examine the in situ response of an MPS layer deposited over an $\mathrm{Al}$ nanocylinder array to the

Received: November 25, 2019

Revised: February 3, 2020 
environmental vapor. We select isopropanol (IPA) as the guest compound that can diffuse and condensate within the pore network of MPS. IPA has been previously proven to yield excellent results for the analysis of the vapor sorption properties of mesostructured and nanoparticle-based multilayers. $^{32}$ In addition, accurate adsorption-desorption isotherms in porous alumina have already been observed and discussed also by using IPA as a probe. ${ }^{33,34}$ The role of MPS as a molecular catcher and condenser has been elucidated experimentally by specular reflectance porosimetry, ${ }^{35,36}$ where the reflectance spectra are measured as a function of the IPA pressure. Compared to the MPS layer on the flat substrate, the MPS layer-on-plasmonic array shows much distinct spectral features assignable to LSPR and hybridized modes. We find the difference in the response between the modes appears in the spectra and relate the difference to the spatial distribution of the light energy associated with each mode.

\section{EXPERIMENTAL SECTION}

Preparation of Al Nanocylinder Arrays. Al nanocylinders arranged in a square lattice with a periodicity of $400 \mathrm{~nm}$ were fabricated by using nanoimprint lithography in combination with reactive ion etching (RIE). The height of the nanocylinders was $200 \mathrm{~nm}$, and their diameter was $200 \mathrm{~nm}$. The fabrication procedure is as follows. First, a resist layer was deposited on a thin film of Al. Then, the surface of the resist was nanostructured by nanoimprint techniques (EntreTM3, Obducat), replicating the surface morphology of a Si mold. The sample was then structured by RIE (RIE-101iPH, Samco). The array structures were examined by using a scanning electron microscope (SU8000, Hitachi).

Preparation of an MPS Layer on the Al Array. The MPS layer was designed to comprise cylindrical pores packed hexagonally with the long axis being aligned parallel to the surface. ${ }^{37}$ First, poly(ethylene glycol)-block-poly(propylene glycol)-block-poly(ethylene glycol), $\mathrm{HO}\left(\mathrm{CH}_{2} \mathrm{CH}_{2} \mathrm{O}\right)_{20^{-}}$ $\left(\mathrm{CH}_{2} \mathrm{CH}\left(\mathrm{CH}_{3}\right) \mathrm{O}\right)_{70}\left(\mathrm{CH}_{2} \mathrm{CH}_{2} \mathrm{O}\right)_{20} \mathrm{H}$ (Pluronic123, $5.50 \mathrm{~g}$ ), was dissolved in ethanol $(20.28 \mathrm{~mL})$. Next, tetraethoxysilane (TEOS, $10.7 \mathrm{~mL}$ ), $0.1 \mathrm{M}$ aqueous solutions of hydrochloric acid $(1.08 \mathrm{~mL})$, water $(9.72 \mathrm{~mL})$, and ethanol $(30.4 \mathrm{~mL})$ were stirred vigorously in another vessel for $20 \mathrm{~min}$. Then, these solutions were mixed and stirred for $3 \mathrm{~h}$. This mixture was used as a precursor solution. The $\mathrm{Al}$ nanocylinder array was dipcoated with the precursor solution to form an MPS layer. After evaporation of the solvent, the sample was heated at a rate of 1 ${ }^{\circ} \mathrm{C} / \mathrm{min}$ to $400{ }^{\circ} \mathrm{C}$ and then held for $4 \mathrm{~h}$ to remove the surfactant inside the pores. The thickness of the MPS layer was evaluated by a stylus profiler (ASIQ KLA Tencor) to be ca. $700 \mathrm{~nm}$.

Specular Reflectance Porosimetry. The specular reflectance was measured under controlled pressure of IPA. To control the vapor pressure in the sample chamber, a container with liquid IPA was connected to the chamber through a needle valve. The internal pressure of the chamber was measured with a dual capacitance manometer (MKS model PDR 2000). Samples were annealed at $200{ }^{\circ} \mathrm{C}$ for $1 \mathrm{~h}$ to remove any moisture from the voids in the porous structures before carrying out the gas adsorption-desorption process. The chamber containing a sample was kept under dynamic vacuum $\left(10^{-2} \mathrm{Torr}\right)$ for $30 \mathrm{~min}$. In adsorption measurements, IPA gas was injected into the chamber, and the reflectance spectra were measured at each fixed pressure, $P$, by using a
FTIR spectrometer (IFS-66, Bruker). This process was repeated sequentially at different vapor pressures until saturation pressure $\left(P_{\mathrm{s}}\right)$ was reached. Desorption experiments were conducted following the same protocol, with a gradual decrease in the pressure being obtained by opening the valve connected to the vacuum pump. Again, spectra were taken at slowly decreasing pressures until the initial value was attained. All measurements were made at room temperature $\left(\sim 20^{\circ} \mathrm{C}\right)$.

Optical Transmission. The zeroth-order optical transmission was measured as a function of angle of incidence, $\theta_{\text {in }}$. For the measurement, we used the collimated beam from a halogen lamp with a beam diameter of ca. $0.5 \mathrm{~mm}$. The sample was mounted on a computer-controlled rotation stage. The absolute zeroth-order (ballistic) transmission as a function of wavelength $\lambda$ and $\theta_{\text {in }}, T\left(\lambda, \theta_{\text {in }}\right)$, was obtained by normalizing the transmission of the incident light through the sample to that of the glass substrate. The incident light was polarized along the $x$-axis (p-polarization), and $\theta_{\text {in }}$ was varied in the $z x$ plane to put momentum into the $x$ direction (see Figure 1a for the coordinate axes). Separately, optical transmittance at $\theta_{\text {in }}=$ $0^{\circ}$ was measured using a UV-visible-near-infrared (UVVis-NIR) spectrophotometer (V770, Jasco).
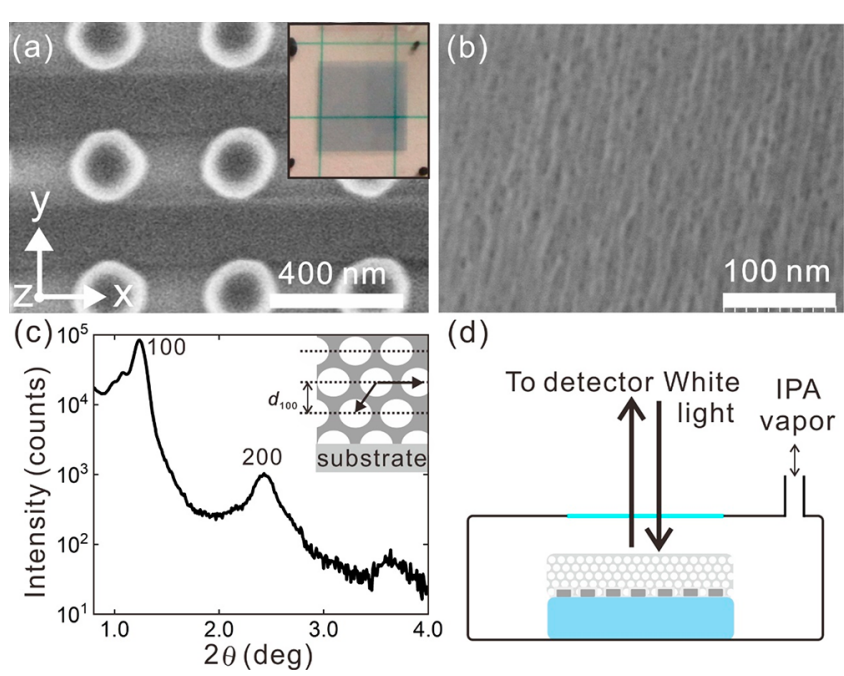

(d)

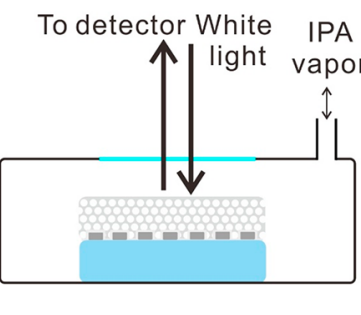

Figure 1. Structural characterization of the MPS layer-on-plasmonic array system. (a) SEM image of the square array of $\mathrm{Al}$ nanocylinders (diameter $200 \mathrm{~nm}$, height $200 \mathrm{~nm}$, array periodicity $400 \times 400 \mathrm{~nm}^{2}$ ). The silica glass substrate is flat, and the horizontal bright and dark lines are due to the charge buildup of the insulating glass substrate. The inset is the photograph of the $6 \mathrm{~mm} \times 6 \mathrm{~mm}$ sized array fabricated on the glass. The $x, y$, and $z$ coordinate axes are also shown. (b) Top-view SEM image of the MPS layer. The thickness of the MPS layer was evaluated by a stylus profiler (KLA Tencor) to be ca. 700 $\mathrm{nm}$. (c) XRD pattern for the MPS layer. XRD peaks come from the hexagonal pack of cylindrical pores lying parallel to the substrate. The inset to (c) is a sketch of the cross-sectional planes of the MPS with the hexagonal vectors used to assign the diffraction peaks. The interplanar distance is calculated to be $d_{100}=7.1 \mathrm{~nm}$. (d) Sketch of the specular reflectance measurement as a function of IPA vapor pressure.

Numerical Simulation. The reflectance for the reference MPS layer was calculated by using an analytical code based on the transfer matrix method (TMM). The reflectance and light energy distribution were simulated using the finite-differential time-domain (FDTD) method (Lumerical). The coordinate axes were set according to the experiment. Two models were 
used to simulate the MPS layer on the flat glass and the MPS layer-on-plasmonic array. From the bottom upward, the following layers were used for the MPS layer on the flat glass: silica substrate (refractive index $n_{\text {sub }}=1.46$ ), the MPS layer $\left(d_{\text {film }}=665 \mathrm{~nm}, n\right.$ was from the TMM fit), and vacuum on the top $\left(n_{\mathrm{vac}}=1.00\right)$. For the MPS layer-on-plasmonic array, we used: silica substrate, the $\mathrm{Al}$ truncated cone (diameter $=200 \mathrm{~nm}, z \operatorname{span}=200 \mathrm{~nm}$, full height $=600 \mathrm{~nm}, n_{\mathrm{Al}}$ was obtained from ref 38$)$, the MPS layer $\left(d_{\text {film }}=730 \mathrm{~nm}, n\right.$ from the TMM fit), and vacuum on the top. A plane wave with the electric field oscillating in the $x$ direction was incident on the top to simulate the transmittance and the spatial distribution of the light energy in the samples. Notice that both TMM and FDTD give the same result for the MPS layer on the flat glass.

\section{RESULTS AND DISCUSSION}

Structural Characterization. Figure 1a shows a top-view SEM image of the $\mathrm{Al}$ nanocylinder array. $\mathrm{Al}$ nanocylinders with diameter $200 \mathrm{~nm}$ are arranged in square pattern with a periodicity of $400 \mathrm{~nm}$. The array exhibits structural color because of light diffraction. Figure $1 \mathrm{~b}$ is a top-view SEM image of the MPS layer. The cylindrical pores are arranged parallel to the surface of the layer with a local alignment. There are additional meso- and micropores that interconnect the cylindrical pores, and thus the film is accessible from the top surface. ${ }^{31}$ Figure 1c illustrates the X-ray diffraction (XRD) pattern measured at small incident angles for the MPS layer on the array to identify the periodicity of the mesoporous structure. The peaks observed at $2 \theta=1.24^{\circ}, 2.43^{\circ}$, and $3.66^{\circ}$ are assigned to the 100,200 , and 300 crystal planes, respectively. The MPS we fabricated is a SBA-15 type, ${ }^{39}$ and these peaks come from the hexagonal pack of cylindrical pores lying parallel to the substrate. The cross section of the pores is elliptic as sketched in the inset of Figure 1c because of the anisotropic shrinkage during the heat treatment. The pores shrink in the out-of-plane direction while in-plane shrinkage is suppressed because of the stress from the substrate. ${ }^{40}$ The interplanar distance is calculated to be $d_{100}=7.1 \mathrm{~nm}$. We have measured the XRD at several azimuthal orientations, and no difference in peak positions was found, indicating the absence of global orientation of domains in the MPS layer; that is, the MPS layer consists of the randomly oriented domains in which the cylindrical pores are aligned in-plane in one direction.

Figure 1d shows the optical setup of the specular reflectance porosimetry measurement. The sample is placed in the chamber under controlled pressure of IPA. The white light impinges on the sample at normal incidence, and the reflected light is analyzed by using Fourier transform spectroscopy (see the Experimental Section for details).

Optical Characterization. To understand the modes excitable in the MPS layer-on-plasmonic array system, optical transmission is examined as a function of angle of incidence $\theta_{\text {in }}$, as shown in Figure 2. The transmission shows a broad dip at the wavelength $\lambda \sim 600 \mathrm{~nm}$, which relates to the excitation of LSPRs of Al nanocylinders. The angular profile of the dip is modulated by several dispersive lines. The dotted lines in the figure are the in-plane diffraction conditions, which are termed Rayleigh anomalies and satisfy the following relation for a square lattice; ${ }^{41} k_{0}^{2}=\left[k_{\|}+m_{1}(2 \pi / a)\right]^{2}+m_{2}^{2}(2 \pi / a)^{2}$, where $k_{0}$ $=2 \pi n_{\text {sur }} / \lambda$ and $k_{\|}=(2 \pi / \lambda) \sin \theta_{\text {in }}$ are the wave vectors of the scattered light and the incident light, respectively, $n_{\text {sur }}$ is the refractive index of the surrounding medium, $a$ is the periodicity of the array, and $m_{1}$ and $m_{2}$ are the diffraction order in the $x$
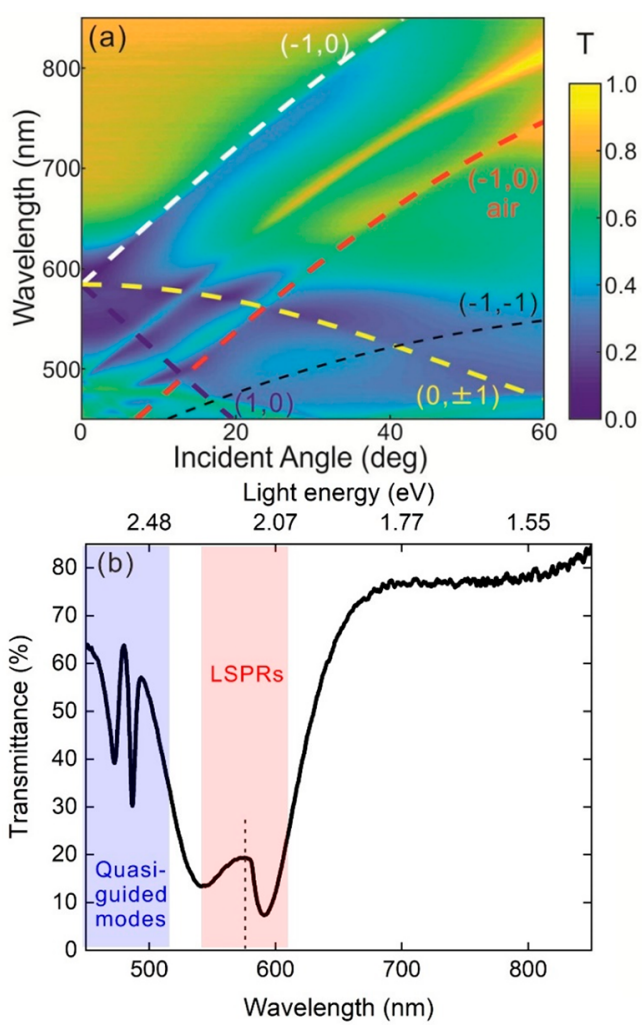

Figure 2. Optical characterization of the MPS layer-on-plasmonic array system measured under atmospheric condition. Transmittance spectra (p-pol) as a function of incident angle. The incidence plane is defined in the $z x$-plane. The zeroth-order transmitted light was collected by a detector (USB4000, Ocean Optics). Rayleigh anomaly conditions are denoted as dotted lines. Unassigned dispersive features are ascribed to quasi-guided modes. (b) A cut of experimental ballistic transmittance spectrum in (a) at the normal incidence. The vertical line indicates the in-plane diffraction condition.

and $y$ directions, respectively. Modulation of LSPRs along the diffraction indicates the simultaneous excitation of LSPRs and in-plane diffraction. It is noted some dispersive features do not follow the diffraction lines. These features come from quasiguided modes, where the MPS layer acts as a waveguide. Figure $2 \mathrm{~b}$ shows the transmission at $\theta_{\text {in }}=0^{\circ}$. The main transmittance dip around $\lambda=600 \mathrm{~nm}$ is the signature of a LSPR split by the in-plane diffraction indicated by the dotted vertical line, and the small dips at shorter wavelengths can be associated with the quasi-guided modes. We focus on these modes in the specular reflectance porosimetry analysis.

Response of the MPS Layer on the Flat Substrate to IPA. Figure 3 summarizes the specular reflectance characterization of the MPS layer on the flat silica glass substrate without the array. Figures $3 a-c$ show the specular reflectance spectra as a function of IPA pressure, represented as IPA pressure $P$ normalized to the saturation vapor pressure of IPA, $P_{s}\left(=4.3 \times 10^{3} \mathrm{~Pa}\right.$ at $\left.20^{\circ} \mathrm{C}\right)$. Spectra show a Fresnel-like fringe pattern due to coherent interference between the reflected light at the interfaces. With the increase in relative pressure in the chamber, gas molecules adsorb onto the pore walls. Eventually, capillary condensation within the pores takes place. Both phenomena lead to an increment in the effective refractive index of the MPS layer, red-shifting the fringes. At a certain relative pressure around $P / P_{s} \sim 0.85$, a notable jump in spectral position is observed. Such an increase corresponds 

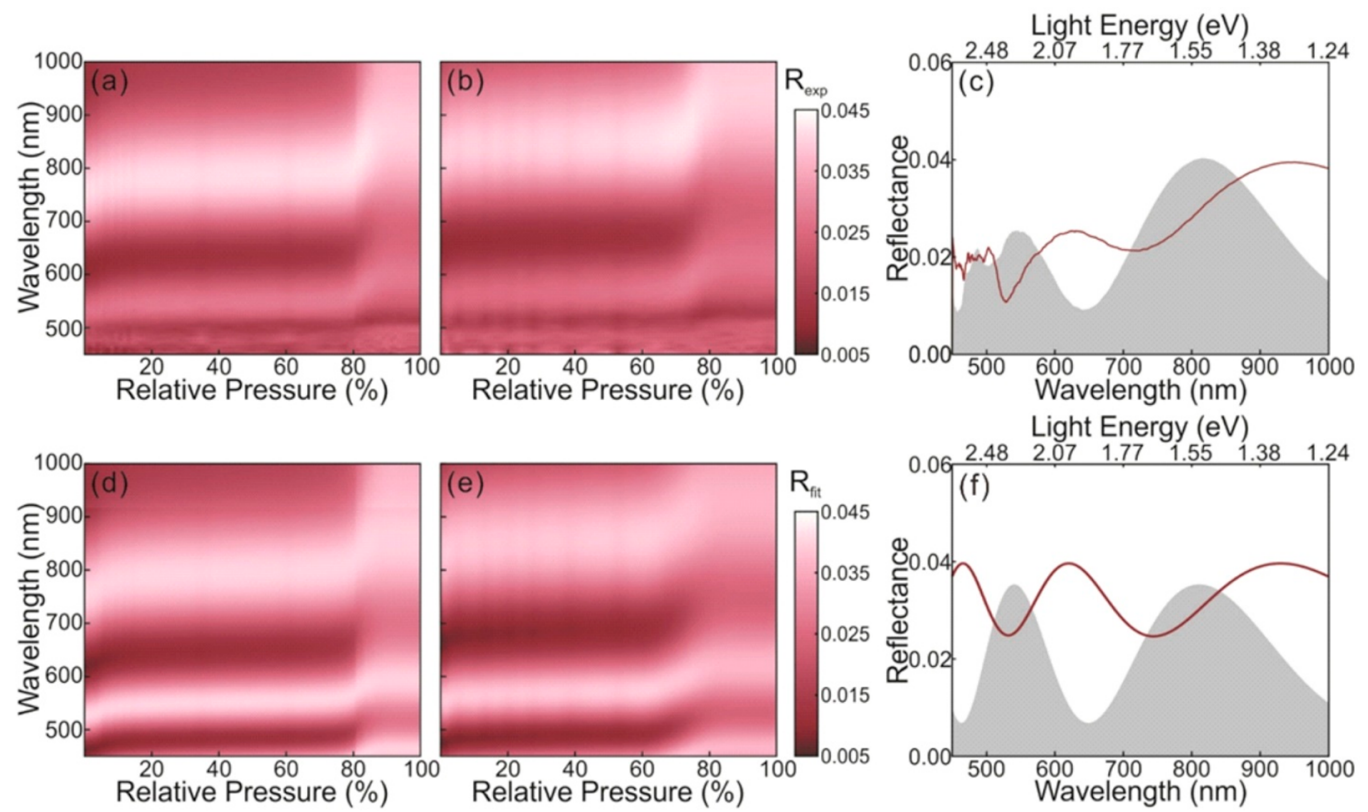

Figure 3. Characterization of the reference MPS layer without nanocylinder array. (a) Experimental specular reflectance spectra with varied IPA pressure for (a) adsorption and (b) desorption processes. (c) Experimental specular reflectance spectra at the initial (relative IPA pressure $=0.001$, represented as gray area) and the highest pressure (relative pressure $=1.0$, maroon line) states. Simulated spectra for $(\mathrm{d})$ adsorption and (e) desorption processes. (f) Simulated specular reflectance spectra at the initial (relative pressure $=0.001$ ) and the highest pressure (relative pressure $=1.0$ ) states in the adsorption process.

to the condensation of IPA inside the pores. In the desorption experiment, the evaporation of IPA from the pores takes place at a lower relative pressure $\left(P / P_{s} \sim 0.75\right)$ than the condensation in the adsorption experiments. This hysteresis appears when the pore has access to the external surface only through a narrower neck, as the cylindrical pores in this study. ${ }^{36}$ The hysteresis is also observed in the $\mathrm{N}_{2}$ adsorption measurement that is the standard technique of pore-sizedistribution analysis. ${ }^{42-45}$ Spectra do not return to the original position even after the pressure reaches the lowest value in the desorption experiment. This means that the molecules attached to the micropores cannot detach. In turn, after a heat treatment at $200{ }^{\circ} \mathrm{C}$ for $1 \mathrm{~h}$, molecules are eventually detached, and the spectra return to the original position. Figure $3 \mathrm{c}$ shows cuts in two-dimensional maps of Figure $3 \mathrm{a}$ at $P / P_{\mathrm{s}}=$ 0.001 and 1.0. The spectrum at $P / P_{\mathrm{s}}=0.001$ shows two broad peaks at $\lambda=525$ and $814 \mathrm{~nm}$. These peaks red-shift when $P / P_{\mathrm{s}}$ $=1.0$ to $\lambda=624$ and $942 \mathrm{~nm}$, respectively, and the shifts are as large as $128 \mathrm{~nm}(0.207 \mathrm{eV})$ for the longer wavelength mode. It is noted that the reflectance is very low, i.e., $\sim 0.04$. Because the MPS layer has the intermediate index of refraction between the air and the glass substrate, it reduces the optical impedance mismatch between them. We simulate the pressure-variant specular reflectance by using an in-house analytical code based on the transfer matrix method. We consider the MPS layer as an effective medium with homogeneous refractive index $n_{\text {eff }}$ and successfully reproduce the pressure-variant specular reflectance as seen in Figures $3 \mathrm{~d}$,e using the dispersive $n_{\text {eff }}$ as a fitting parameter (the value of $n_{\text {eff }}$ is shown in Figure S1). Figure $3 \mathrm{f}$ displays the spectra at $P / P_{s}=0.001$ and 1.0 in the adsorption process. Nearly quantitative agreement is obtained with the experimental reflectance in Figure 3c. Pressure- and wavelength-dependent $n_{\text {eff }}$ of the effective medium is further used to simulate the MPS layer-on-plasmonic array system, as it will be shown in the next section.
We analyze the pore size distribution based on the Brunauer-Emmett-Teller (BET) equation following a protocol in ref32 (see Figure S2 for details). The mean pore radii are 7.8 and $5.2 \mathrm{~nm}$ from adsorption and desorption curves, respectively. The pore sizes estimated from the BET equation are on the same order but larger than that from SEM (Figure 1b) and XRD (Figure 1c). This is because of the discrepancy in pore shape between the sample and the model. We analyze the data using the spherical pore model with a single curvature for the sake of simplicity. However, the MPS layer consists of cylindrical pores with elliptic cross section where the curvature in one dimension is zero. The discrepancy may lead to overestimation of the effective pore size.

Response of the MPS Layer-on-Plasmonic Array System to IPA. Figure 4 shows the experimental specular reflectance spectra for the MPS layer-on-plasmonic array system as a function of $P / P_{s}$. The spectral features red-shift as $P / P_{s}$ increases. It is noticed that the jump in the shift occurs at $P / P_{s} \sim 0.85$ and 0.75 in the adsorption and desorption, respectively, corresponding to the condensation of the IPA molecules in the pores. These relative pressures are the same as that for the reference MPS layer, meaning that the pore characteristics of the layers on the array and on the flat substrate are identical to each other. Figure $4 c$ shows the cuts in two-dimensional maps of Figure $4 \mathrm{a}$ at $P / P_{s}=0.002$ and 1.0. The shift in the peaks is noticeable. Here we pick three reflectance peaks from different origins, indicated by arrows, and compare the shifts, as summarized in Table 1 . It can be noticed that the Fresnel mode $\left(\lambda=746 \mathrm{~nm}\right.$ at $\left.P / P_{s}=0.002\right)$ features the largest shift value in terms of wavelength, whereas it is the quasi-guided mode $\left(\lambda=505 \mathrm{~nm}\right.$ at $\left.P / P_{\mathrm{s}}=0.002\right)$ in terms of energy shift. The $\operatorname{LSPR}\left(\lambda=601 \mathrm{~nm}\right.$ at $\left.P / P_{s}=0.002\right)$ shows the smallest shift. This different sensitivity is discussed in the next section. 

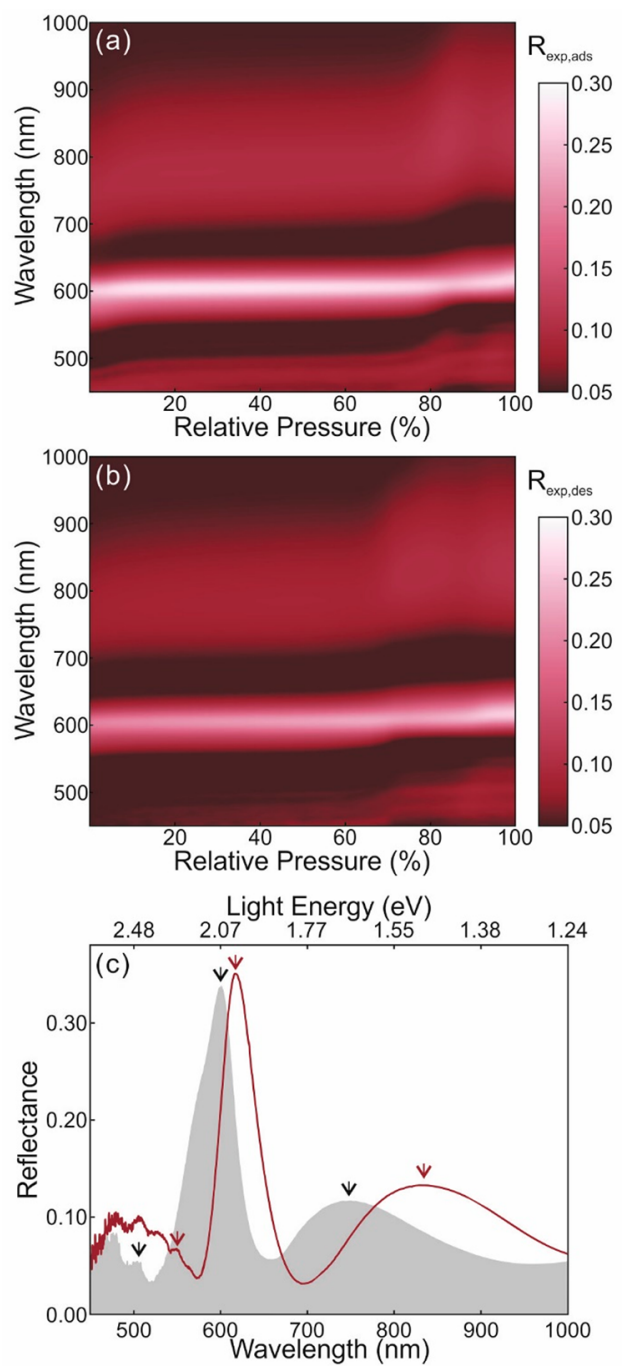

Figure 4. Experimental specular reflectance spectra for the MPS layeron-plasmonic array system with varied IPA pressure: (a) adsorption and (b) desorption processes. (c) Specular reflectance spectra at the initial (relative pressure $=0.002$, represented as gray area) and the highest pressure (relative pressure $=1.0$, maroon line) states. The arrows indicate the spectral peak positions focused in this study.

Table 1. Comparison of the Spectral Positions of Reflectance Peaks at $P / P_{s}=0.002$ and 1.0 and the Shift Values $^{a}$

\begin{tabular}{lccc}
\multicolumn{1}{c}{ mode } & $P / P_{\mathrm{s}}=0.002(\mathrm{~nm} / \mathrm{eV})$ & $P / P_{\mathrm{s}}=1.0(\mathrm{~nm} / \mathrm{eV})$ & $\begin{array}{c}|\Delta| \\
(\mathrm{nm} / \mathrm{eV})\end{array}$ \\
quasi-guided & $505 / 2.455$ & $551 / 2.250$ & $46 / 0.205$ \\
LSPR & $601 / 2.063$ & $618 / 2.006$ & $17 / 0.057$ \\
Fresnel & $746 / 1.662$ & $827 / 1.499$ & $81 / 0.163$
\end{tabular}

${ }^{a}$ The values are extracted from the data of adsorption (Figure 4) for the MPS layer-on-plasmonic array system.

The condensation allows for the detection of low vapor pressure of IPA. The spectral shift at $P / P_{\mathrm{s}}<1.0$, where $P_{\mathrm{s}}=4.3$ $\times 10^{3} \mathrm{~Pa}$ at $20{ }^{\circ} \mathrm{C}$, indicates that IPA vapor with a concentration $<2 \mu \mathrm{mol} \mathrm{cm}^{-3}$ can be detected under atmospheric conditions. The detection limit is further reduced as low as $20 \mathrm{nmol} \mathrm{cm} \mathrm{cm}^{-3}$ considering the reflectance change occurs at $P / P_{\mathrm{s}}<0.01$. This value is smaller than the other vapor-sensing systems ${ }^{15,26}$ and is achieved because the mesopores in MPS layers are small enough to condensate IPA vapor at low pressures.

Mode Extension and the Sensitivity. Figure 5 shows the simulated reflectance as a function of $P / P_{s}$. Here we calculate the spectra using $n_{\text {eff }}$ deduced from the simulation for the reference (see Figure S1). Comparison between Figures $4 a-c$ and $5 \mathrm{a}-\mathrm{c}$ shows that the agreement between the experiment and the simulation is good. This confirms that the adsorptiondesorption properties of the MPS layer-on-plasmonic array are the same as those on the flat substrate. A slight difference appears in experimental and simulated reflectance spectra in Figures $4 \mathrm{c}$ and $5 \mathrm{c}$ where the simulation shows a splitting of LSPR. This comes from the difference that in experiment illumination and collection are through the objective lens (numerical aperture $=0.1$ ) while the simulation is for normal incidence.

To understand the difference in the spectral shift of the reflectance peaks, we simulate the light energy distributions inside the system upon irradiation with plane lightwaves at three typical wavelengths for $P / P_{s}=0.002$. For the quasiguided mode at $\lambda=461 \mathrm{~nm}$ (Figure $5 \mathrm{~d}$ ), the light energy is distributed in both inside the MPS layer and in the vicinity of the $\mathrm{Al}$ nanocylinder, reflecting the hybrid nature of the mode between the LSPR and the waveguide. For the LSPR at $\lambda=$ $598 \mathrm{~nm}$ (Figure 5e), the energy localized in the vicinity of the nanocylinder is the largest among the three wavelengths, resulting in the largest reflectance intensity among them. Even for the Fresnel mode at $\lambda=755 \mathrm{~nm}$ (Figure 5f), the light energy is not only distributed to the MPS layer but also confined around the nanocylinder. This increases the reflectance intensity compared to the Fresnel modes that appear for the MPS layer on the flat substrate, where the highest reflectance is only $\sim 0.04$.

Because the relative IPA pressure changes the effective refractive index of the MPS layer, the large distribution of the light energy in the layer results in the large shift of the peak; that is, the modes with the light energy extended into the layer are more sensitive compared to the modes localized in the nanocylinder. This explains the smallest shift for the LSPR mode among the three. The Fresnel modes that are observed for the MPS layer on the flat substrate in Figure $3 \mathrm{c}$ show the shift larger than any of the modes in the layer on the array because it is extended in the layer. However, the intensity of the peak is very low because of the small refractive index mismatch at the interface. The simulation (Figure $5 \mathrm{~g}$ ) confirms the extended but notably small light energy distribution in the layer. The reflectance of MPS layer-on-plasmonic array system is larger because of the partial hybridization with LSPRs even for the Fresnel mode as shown in the spatial field distribution.

The benefit of the MPS layer-on-plasmonic array system is emphasized by the comparison to the "MPS layer-on-mirror" system where the MPS layer is on a metallic film. Our simulations show a similar optical behavior when comparing the reflectance spectrum of the porous layer deposited over a glass substrate or a metallic film. Indeed, the width and depth of Fresnel-type reflectance fringes along with the spectral shift of these modes with the refractive index change are on the same order regardless of the substrate they are deposited (see Figure S3). Only absolute reflectance values are larger when the porous layer is deposited over the $\mathrm{Al} \mathrm{film}$, which acts as a mirror. In contrast, the plasmonic array provides similar values of the spectral shift with the refractive index but significantly larger reflectance contrast. Consequently, the MPS layer-on- 

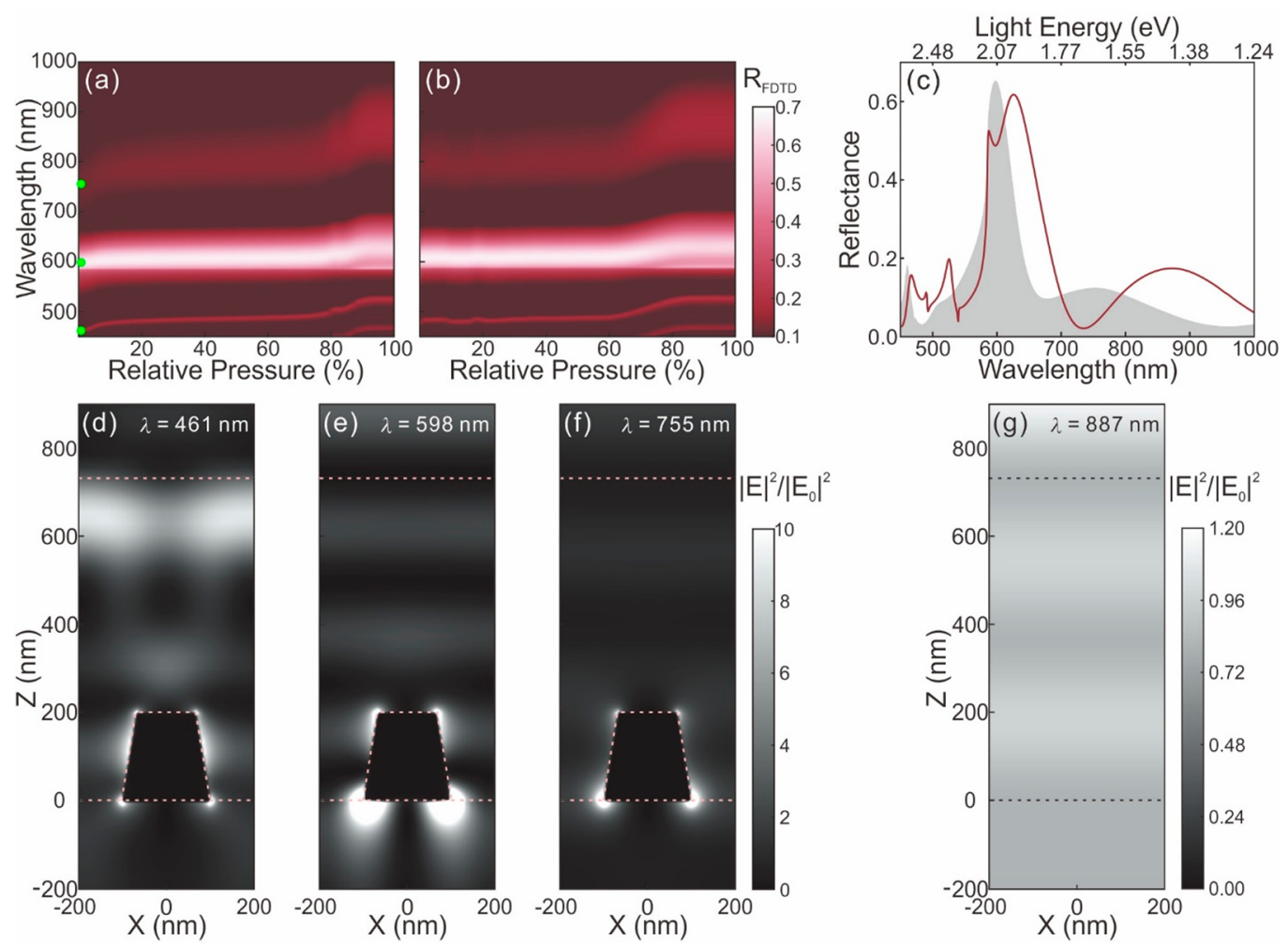

Figure 5. Simulated specular reflectance spectra for the MPS layer on the array of Al nanocylinders as a function of IPA relative pressure: (a) adsorption and (b) desorption processes. The effective refractive index values of the MPS layer are extracted from the fit to the specular reflectance for the reference layer without the array (Figure S1). The circles denote the calculation conditions of spatial energy distribution in (d-f). (c) Simulated specular reflectance spectra at the initial (relative pressure $=0.002$, represented as gray area) and the highest pressure (relative pressure $=$ 1.0, maroon line) states in the adsorption process. $(\mathrm{d}-\mathrm{g})$ Calculated spatial distributions of the squared magnitude of the electric field normalized to the incident field, $|E|^{2} /\left|E_{0}\right|^{2}$, in the $z x$-plane, at a $y$ position intersecting the middle of a nanocylinder (relative pressure $\left.=0.002\right)$. Dashed lines represent the boundaries of the materials. The wavelength of the incident light (polarized along $x$-axis) is $\lambda=(\mathrm{d}) 461$, (e) 598, and (f) $755 \mathrm{~nm}$. Calculated spatial distribution of light energy for the reference MPS layer upon irradiation with $\lambda=887 \mathrm{~nm}$ plane wave is plotted in (g).

plasmonic array system shows large reflection contrast and an acute optical response to chemicals that is visible to the eyes (see Figure S4), which are beneficial for application as optical environmental sensors.

\section{CONCLUSIONS}

We made a plasmonic system that responds to the relative pressure of IPA. This was achieved by covering the array of $\mathrm{Al}$ nanocylinders with a slab waveguide layer of MPS in which the mesosized pores act as catcher and condenser of the IPA molecules. We characterized the response by using specular porosimetry, where the reflectance peaks shifted with the relative pressure of IPA. The shift depends on the mode extension in the MPS layer, while the intensity of the reflectance peak depends mainly on the strength of the interaction between incident lightwave with the LSPRs. The combination of the MPS layer with the Al nanocylinder array achieves both extension of the mode inside the layer and the strong interaction, which are useful for optical sensors.

\section{ASSOCIATED CONTENT}

\section{SI Supporting Information}

The Supporting Information is available free of charge at https://pubs.acs.org/doi/10.1021/acs.jpcc.9b10999.

Characterization of the reference MPS layer without nanocylinder array, reflection of MPS layer-on-mirror system, color change of MPS layer-on-plasmonic array system (PDF)

\section{AUTHOR INFORMATION}

Corresponding Authors

Shunsuke Murai - Department of Material Chemistry, Graduate School of Engineering, Kyoto University, Kyoto 6158510, Japan; ○orcid.org/0000-0002-4597-973X;

Phone: +81-75-383-2422; Email: murai@

dipole7.kuic.kyoto-u.ac.jp

Gabriel Lozano - Consejo Superior de Investigaciones Cientificas-Universidad de Sevilla, Instituto de Ciencia de Materiales de Sevilla, 41092 Sevilla, Spain; (1) orcid.org/00000002-0235-4924; Phone: +34-95-448-9500;

Email: glozano@icmse.csic.es

\section{Authors}

Elena Cabello-Olmo - Consejo Superior de Investigaciones Cientificas-Universidad de Sevilla, Instituto de Ciencia de Materiales de Sevilla, 41092 Sevilla, Spain; (1) orcid.org/00000002-3921-6605

Ryosuke Kamakura - Department of Material Chemistry, Graduate School of Engineering, Kyoto University, Kyoto 6158510, Japan; 이이이.org/0000-0002-5859-6850

Mauricio E. Calvo - Consejo Superior de Investigaciones Cientificas-Universidad de Sevilla, Instituto de Ciencia de Materiales de Sevilla, 41092 Sevilla, Spain; (1) orcid.org/00000002-1721-7260 
Taisuke Atsumi - Department of Material Chemistry, Graduate School of Engineering, Kyoto University, Kyoto 615-8510, Japan

Hernán Míguez - Consejo Superior de Investigaciones Cientificas-Universidad de Sevilla, Instituto de Ciencia de Materiales de Sevilla, 41092 Sevilla, Spain; (ㅇ orcid.org/00000003-2925-6360

Katsuhisa Tanaka - Department of Material Chemistry, Graduate School of Engineering, Kyoto University, Kyoto 6158510, Japan; 이이이.org/0000-0002-1409-2802

Complete contact information is available at:

https://pubs.acs.org/10.1021/acs.jpcc.9b10999

\section{Author Contributions}

S.M. and E.C.-O. contributed equally to this work.

\section{Notes}

The authors declare no competing financial interest.

\section{ACKNOWLEDGMENTS}

This work was partly supported by Grant-in-Aids for Ministry of Education, Culture, Sports, Science and Technology (16H04217, 19H02434, Nanotech CUPAL); the Japan Science and Technology Agency (PRESTO, No. JPMJPR131B); The Asahi Glass Foundation; Nanotechnology Hub, Kyoto University, the National Institute for Material Science (NIMS) Nanofabrication Platform; the European Research Council (ERC); the European Union's Horizon 2020 research and innovation programme (NANOPHOM, grant agreement no. 715832); and the Spanish Ministry of Economy and Competitiveness under grant MAT2017-88584-R.

\section{REFERENCES}

(1) Ballato, J.; James, A. A ceramic photonic crystal temperature sensor. J. Am. Ceram. Soc. 1999, 82, 2273-2275.

(2) Murai, S.; Yao, S.; Nakamura, T.; Kawamoto, T.; Fujita, K.; Yano, K.; Tanaka, K. Modified Faraday rotation in a threedimensional magnetophotonic opal crystal consisting of maghemite/ silica composite spheres. Appl. Phys. Lett. 2012, 101, 151121-1-4.

(3) Sanchez-Sobrado, O.; Lozano, G.; Calvo, M. E.; Sanchez-Iglesias, A.; Liz-Marzan, L. M.; Miguez, H. Interplay of resonant cavity modes with localized surface plasmons: Optical absorption properties of bragg stacks integrating gold nanoparticles. Adv. Mater. 2011, 23, $2108-12$.

(4) Meng, X.; Kildishev, A. V.; Fujita, K.; Tanaka, K.; Shalaev, V. M. Wavelength-tunable spasing in the visible. Nano Lett. 2013, 13, 410612.

(5) Sanchez-Sobrado, O.; Calvo, M. E.; Nunez, N.; Ocana, M.; Lozano, G.; Miguez, H. Environmentally responsive nanoparticlebased luminescent optical resonators. Nanoscale 2010, 2, 936-941.

(6) Kang, D.; Maclennan, J. E.; Clark, N. A.; Zakhidov, A. A.; Baughman, R. H. Electro-optic behavior of liquid-crystal-filled silica opal photonic crystals: Effect of liquid-crystal alignment. Phys. Rev. Lett. 2001, 86, 4052-4055.

(7) Mertens, G.; Roder, T.; Schweins, R.; Huber, K.; Kitzerow, H. S. Shift of the photonic band gap in two photonic crystal/liquid crystal composites. Appl. Phys. Lett. 2002, 80, 1885-1887.

(8) Murai, S.; Fujita, K.; Hirao, T.; Nakanishi, K.; Hirao, K. Temperature-tunable scattering strength based on the phase transition of liquid crystal infiltrated in well-defined macroporous random media. Opt. Mater. 2007, 29, 949-954.

(9) Colodrero, S.; Ocaña, M.; González-Elipe, A. R.; Míguez, H. Response of nanoparticle-based one-dimensional photonic crystals to ambient vapor pressure. Langmuir 2008, 24, 9135-9139.

(10) Bonifacio, L. D.; Puzzo, D. P.; Breslav, S.; Willey, B. M.; McGeer, A.; Ozin, G. A. Towards the photonic nose: A novel platform for molecule and bacteria identification. Adv. Mater. 2010, $22,1351-1354$

(11) Szendrei, K.; Ganter, P.; Sànchez-Sobrado, O.; Eger, R.; Kuhn, A.; Lotsch, B. V. Touchless optical finger motion tracking based on $2 \mathrm{~d}$ nanosheets with giant moisture responsiveness. Adv. Mater. 2015, 27, 6341-6348.

(12) Szendrei, K.; Jiménez-Solano, A.; Lozano, G.; Lotsch, B. V.; Míguez, H. Fluorescent humidity sensors based on photonic resonators. Adv. Opt. Mater. 2017, 5, 1700663.

(13) Yamada, Y.; Nakamura, T.; Ishi, M.; Yano, K. Reversible control of light reflection of a colloidal crystal film fabricated from monodisperse mesoporous silica spheres. Langmuir 2006, 22, 24446.

(14) Yamada, Y.; Nakamura, T.; Yano, K. Optical response of mesoporous synthetic opals to the adsorption of chemical species. Langmuir 2008, 24, 2779-84.

(15) Liu, J.; Zhang, Y.; Zhou, R.; Gao, L. Volatile alcohol-responsive visual sensors based on $\mathrm{p}$ (HEMA-co-MA)-infiltrated $\mathrm{SiO}_{2}$ inverse opal photonic crystals. J. Mater. Chem. C 2017, 5, 6071-6078.

(16) Barnes, W. L.; Dereux, A.; Ebbesen, T. W. Surface plasmon subwavelength optics. Nature 2003, 424, 824-30.

(17) Malmqvist, M. Surface plasmon resonance for detection and measurement of antibody-antigen affinity and kinetics. Curr. Opin. Immunol. 1993, 5, 282-6.

(18) Jing, J.-Y.; Wang, Q.; Zhao, W.-M.; Wang, B.-T. Long-range surface plasmon resonance and its sensing applications: A review. Opt. Laser Eng. 2019, 112, 103-118.

(19) Cao, C.; Sim, S. J. Signal enhancement of surface plasmon resonance immunoassay using enzyme precipitation-functionalized gold nanoparticles: A femto molar level measurement of anti-glutamic acid decarboxylase antibody. Biosens. Bioelectron. 2007, 22, 1874-80. (20) Hoang, C. V.; Oyama, M.; Saito, O.; Aono, M.; Nagao, T. Monitoring the presence of ionic mercury in environmental water by plasmon-enhanced infrared spectroscopy. Sci. Rep. 2013, 3, 1175.

(21) Kumar, J.; Liz-Marzán, L. M. Recent advances in chiral plasmonics - towards biomedical applications. Bull. Chem. Soc. Jpn. 2019, 92, 30-37.

(22) Nylander, C.; Liedberg, B.; Lind, T. Gas detection by means of surface plasmon resonance. Sens. Actuators 1982, 3, 79-88.

(23) Abdelghani, A.; Chovelon, J. M.; Jaffrezic-Renault, N.; Veilla, C.; Gagnaire, H. Chemical vapour sensing by surface plasmon resonance optical fibre sensor coated with fluoropolymer. Anal. Chim. Acta 1997, 337, 225-232.

(24) Arakawa, T.; Kawabayashi, A.; Saga, T. Detection of butylamine by means of surface plasmon resonance. Sens. Actuators, B 2005, 108, 899-902.

(25) Wu, L.; Chu, H. S.; Koh, W. S.; Li, E. P. Highly sensitive graphene biosensors based on surface plasmon resonance. Opt. Express 2010, 18, 14395-14400.

(26) Bingham, J. M.; Anker, J. N.; Kreno, L. E.; Van Duyne, R. P. Gas sensing with high-resolution localized surface plasmon resonance spectroscopy. J. Am. Chem. Soc. 2010, 132, 17358-17359.

(27) Mishra, S. K.; Kumari, D.; Gupta, B. D. Surface plasmon resonance based fiber optic ammonia gas sensor using ito and polyaniline. Sens. Actuators, B 2012, 171-172, 976-983.

(28) Maharana, P. K.; Jha, R.; Padhy, P. On the electric field enhancement and performance of spr gas sensor based on graphene for visible and near infrared. Sens. Actuators, B 2015, 207, 117-122.

(29) Usman, F.; Dennis, J. O.; Seong, K. C.; Ahmed, A. Y.; Ferrell, T. L.; Fen, Y. W.; Sadrolhosseini, A. R.; Ayodele, O. B.; Meriaudeau, F.; Saidu, A. Enhanced sensitivity of surface plasmon resonance biosensor functionalized with doped polyaniline composites for the detection of low-concentration acetone vapour. J. Sens. 2019, 2019, 1. (30) Zhang, Z.; Lu, D. F.; Liu, Q.; Qi, Z. M.; Yang, L.; Liu, J. Wavelength-interrogated surface plasmon resonance sensor with mesoporous-silica-film-enhanced sensitivity to small molecules. Analyst 2012, 137, 4822-8. 
(31) Murai, S.; Sakamoto, H.; Fujita, K.; Tanaka, K. Mesoporous silica layer on plasmonic array: Light trapping in a layer with a variable index of refraction. Opt. Mater. Express 2016, 6, 2736-2744.

(32) Hidalgo, N.; Lopez-Lopez, C.; Lozano, G.; Calvo, M. E.; Miguez, H. Characterization of mesoporous thin films by specular reflectance porosimetry. Langmuir 2012, 28, 13777-82.

(33) Casanova, F.; Chiang, C. E.; Li, C. P.; Schuller, I. K. Direct observation of cooperative effects in capillary condensation: The hysteretic origin. Appl. Phys. Lett. 2007, 91, 243103.

(34) Casanova, F.; Chiang, C. E.; Li, C. P.; Roshchin, I. V.; Ruminski, A. M.; Sailor, M. J.; Schuller, I. K. Effect of surface interactions on the hysteresis of capillary condensation in nanopores. Europhys. Lett. 2008, 81, 26003.

(35) Baklanov, M. R.; Mogilnikov, K. P.; Polovinkin, V. G.; Dultsev, F. N. Determination of pore size distribution in thin films by ellipsometric porosimetry. J. Vac. Sci. Technol., B: Microelectron. Process. Phenom. 2000, 18, 1385-1391.

(36) Boissiere, C.; Grosso, D.; Lepoutre, S.; Nicole, L.; Bruneau, A. B.; Sanchez, C. Porosity and mechanical properties of mesoporous thin films assessed by environmental ellipsometric porosimetry. Langmuir 2005, 21, 12362-12371.

(37) Zhao, D.; Yang, P.; Melosh, N.; Feng, J.; Chmelka, B. F.; Stucky, G. D. Continuous mesoporous silica films with highly ordered large pore structures. Adv. Mater. 1998, 10, 1380-1385.

(38) Palik, E. Handbook of optical constants of solids. 1985; Vol. 1.

(39) Zhao, D.; Feng, J.; Huo, Q.; Melosh, N.; Fredrickson, G. H.; Chmelka, B. F.; Stucky, G. D. Triblock copolymer syntheses of mesoporous silica with periodic 50 to 300 angstrom pores. Science 1998, 279, 548-552.

(40) Hayase, S.; Kanno, Y.; Watanabe, M.; Takahashi, M.; Kuroda, K.; Miyata, H. Heteroepitaxial formation of aligned mesostructured silica films with large structural periodicities from mixed surfactant systems. Langmuir 2013, 29, 7096-7101.

(41) Vecchi, G.; Giannini, V.; Gómez Rivas, J. Surface modes in plasmonic crystals induced by diffractive coupling of nanoantennas. Phys. Rev. B: Condens. Matter Mater. Phys. 2009, 80, 201401.

(42) Thommes, M.; Smarsly, B.; Groenewolt, M.; Ravikovitch, P. I.; Neimark, A. V. Adsorption hysteresis of nitrogen and argon in pore networks and characterization of novel micro- and mesoporous silicas. Langmuir 2006, 22, 756-764.

(43) Esparza, J. M.; Ojeda, M. L.; Campero, A.; Domínguez, A.; Kornhauser, I.; Rojas, F.; Vidales, A. M.; López, R. H.; Zgrablich, G. $\mathrm{N}_{2}$ sorption scanning behavior of SBA-15 porous substrates. Colloids Surf., A 2004, 241, 35-45.

(44) Morishige, K.; Ito, M. Capillary condensation of nitrogen in MCM-41 and SBA-15. J. Chem. Phys. 2002, 117, 8036-8041.

(45) Inagaki, S.; Fukushima, Y.; Kuroda, K.; Kuroda, K. Adsorption isotherm of water vapor and its large hysteresis on highly ordered mesoporous silica. J. Colloid Interface Sci. 1996, 180, 623-624. 